\title{
USO DO KAHOOT COMO FERRAMENTA DE AVALIAÇÃO E ENSINO-APRENDIZAGEM NO ENSINO DE MICROBIOLOGIA INDUSTRIAL
}

\author{
D. SANDE ${ }^{1 *}$, D. SANDE ${ }^{2}$ \\ ${ }^{1}$ Universidade Federal de Minas Gerais, ${ }^{2}$ Universidade Salvador \\ sandebiom@gmail.com*
}

Submetido 17/09/2017 - Aceito 31/01/2018

DOI: $10.15628 /$ holos. 2018.6300

\section{RESUMO}

A gamificação é uma estratégia de usar elementos dos jogos fora do ambiente dos jogos e pode ser aplicada para auxiliar o professor na tarefa não mais de fornecer informações mas sim de ensinar a selecionar as informações úteis e de aplicar esse conhecimento para resolver problemas da profissão. Nesse contexto, foi aplicado um quiz usando a plataforma kahoot como estratégia de avaliação e ensino-aprendizagem na disciplina Microbiologia Industrial. Os alunos realizaram a atividade avaliativa e em seguida responderam a um questionário através de um formulário do GoogleDocs. Os alunos citaram a competição como estímulo para o aprendizado e perceberam que o kahoot pode ser usado como substituto da avaliação tradicional uma vez que consegue alcançar diferentes níveis de complexidade que desafiam os alunos e tornam o aprendizado mais eficiente e duradouro. A técnica, entretanto, apresenta algumas limitações. A percepção e interesse dos alunos foi positiva diante da experiência.

PALAVRAS-CHAVE: Metodologia ativa, quiz, avaliação.

\section{KAHOOT AS EVALUATION TOOL AND TEACHING-LEARNING IN THE DISCIPLINE INDUSTRIAL MICROBIOLOGY}

\begin{abstract}
The gamification is a strategy to use elements of the games outside the environment of games and can be applied to assist the teacher in the task of providing information but rather to teach to select relevant information and to apply this knowledge to solve problems of profession. In this context, it was applied a quiz using the kahoot platform as evaluation strategy and teaching-learning in the discipline Industrial Microbiology. The students performed the evaluative
\end{abstract}

activity and then responded to a questionnaire through a form of GoogleDocs. Students cite competition as stimulus for learning and they realized the kahoot can be used as a replacement for traditional assessment once you can reach different levels of complexity that challenge students and make learning more efficient and durable. The technique, however, presents some limitations. The perception and interests of students was positive from the experience.

KEY-WORDS: Active methodology, quiz, evaluation. 


\section{INTRODUÇÃO}

Quando a "geração digital" (indivíduos nascidos entre 1982 e 2002) chega à universidade e percebe que tem mais capacidade de aprender acessando um site de busca do que prestando atenção à aula expositiva de um professor ou lendo um livro impresso (Van Nuland et al., 2015), é preciso remodelar a educação!

Quando o conhecimento tinha acesso restrito e os professores eram detentores exclusivos do conhecimento, o foco da educação era passar o máximo de informação possível aos alunos (Miltre et al., 2008). Agora, os alunos que pertencem à "geração digital" têm fácil acesso a smartphones, tablets, computadores e notebooks conectados à internet (Bottentuit Júnior, 2012). Na internet, a informação de diferentes áreas do conhecimento está a poucos cliques de distância (Martinez et al., 2008).

O aluno já percebeu que a informação está disponível, então ele não precisa do professor para isso. Nesse contexto, professores que continuam entregando aulas com único intuito de transmitir o conhecimento integral, estão encontrando alunos desinteressados, desatentos e desmotivados, que não enxergam o propósito de ir à aula (Tardif, 2011).

Diante desse contexto, o foco da educação precisa ser modificado, pois, uma vez que o conhecimento está facilmente acessível, a maior dificuldade dos alunos agora consiste em avaliar criticamente a informação disponível, selecionar o que é útil e entender como ele pode aplicar aquele conhecimento na resolução de problemas do exercício da sua profissão (Miltre et al., 2008; Costa et al., 2017). Nesse ponto, o professor demonstra a sua importância através da sua experiência nesse processo de usar o conhecimento para resolver problemas da prática.

Uma das estratégias para vencer essa barreira que a educação vem enfrentando é promover uma organização de sala de aula invertida, com a aquisição de conhecimentos através de vídeo aulas e materiais digitais em casa, e com a resolução de problemas usando mídias digitais e gamificação na sala de aula. A gamificação consiste no uso de elementos dos jogos, fora do contexto dos jogos, como na educação, por exemplo (Coli et al., 2017) e permite agregar valor às aulas, proporcionando desafio, prazer e entretenimento à transmissão do conhecimento.

Diferentes autores têm publicado experiências com criação e aplicação de jogos educacionais bem como usando a gamificação. Coli et al. (2017), por exemplo, relataram a criação de um jogo de tabuleiro de microbiologia (Gut Checker: the microbiome game) em que conceitos científicos importantes são apresentados de uma forma lúdica e divertida. Martinez et al. (2008), também relatou a criação de um jogo (Um show de genética) que propõe apresentar conteúdos com grande nível de dificuldade usando o entretenimento. Costa et al. (2017), usou ferramentas colaborativas como o Marvinsketch e kahoot, com intuito lúdico, para facilitar a abstração dos alunos na visualização de estrutura tridimensional de moléculas em química orgânica.

Uma vez que o sistema de avaliação também pode ser usado como um momento de ensino aprendizagem (Cavalcanti \& Soares, 2009; Dellos, 2015), esses jogos também podem ser aplicados como estratégias de avaliação, uma vez que possuem mecanismos para o professor acompanhar e avaliar o desempenho dos alunos. Cavalcanti \& Soares (2009), aplicaram RPG (roleplaying games) como estratégia de avaliação do conteúdo de química e obtiveram bons 
resultados, inclusive com intervenção e correção imediata de conceitos durante a aplicação do jogo.

O kahoot é uma plataforma de aprendizado baseada em jogos de diferentes modalidades, incluído um quiz game disponível no site https://kahoot.com/, no qual podem ser adicionadas perguntas pelo professor e, essas são convertidas em um jogo com pontuação, interação e ranqueamento (Dellos, 2015; Costa et al., 2017). Essa ferramenta permite ao professor a criação de vários jogos usando o conteúdo de uma disciplina, bem como permite a avaliação do desempenho do aluno.

O presente trabalho propõe a aplicação de um jogo de quiz, usando a plataforma do kahoot, como estratégia de avaliação de desempenho e de ensino-aprendizagem dos alunos na disciplina microbiologia industrial, dentro do curso de Farmácia.

\section{METOdOLOGIA}

\subsection{A criação do jogo}

A primeira etapa consistiu em preparar o quiz on line utilizando o site https://kahoot.com/ para substituir uma prova escrita da disciplina Microbiologia Industrial, aplicada dentro do curso de Farmácia da universidade Federal de Minas Gerais. Inicialmente realizou-se o cadastro conforme as instruções do site. Em seguida selecionou-se a modalidade "quiz" do kahoot e seguiu-se as instruções de preenchimento do site para a criação quiz. Esse foi intitulado PROVAQUIZ de Micro Industrial conforme pode ser visto na Figura 1 e no link https://create.kahoot.it/\#quiz/700c27e3-597c-42cb-8137-88f476be77c5. Esse quiz foi criado com 25 questões de múltipla escolha (com 3 alternativas incorretas e uma correta) sobre 0 conteúdo ministrado na disciplina e envolvendo tópicos como: Processo biotecnológico industrial genérico; Microrganismos e meios de cultura para utilização industrial; Fermentação em estado sólido; Reatores com células e enzimas imobilizadas; Classificação das fermentações segundo o modo de condução do processo fermentativo; Produtos obtidos por fermentação alcoólica, láctica e outras; Cinética dos processos fermentativos descontínuos; Purificação de biomoléculas. Nas 25 questões criadas foram inseridas 8 figuras, entre criação própria e aquelas retiradas do site do "google imagens", que se repetiam nas questões. Haviam figuras não relacionadas ao tema com referências a desenho animado e figuras técnicas, com referência ao conteúdo estudado. Na criação do jogo também foram definidos os tempos para a resposta de cada questão conforme a dificuldade de cada questão. O tempo mínimo selecionado foi de 60 segundos e o máximo de 120 segundos. 


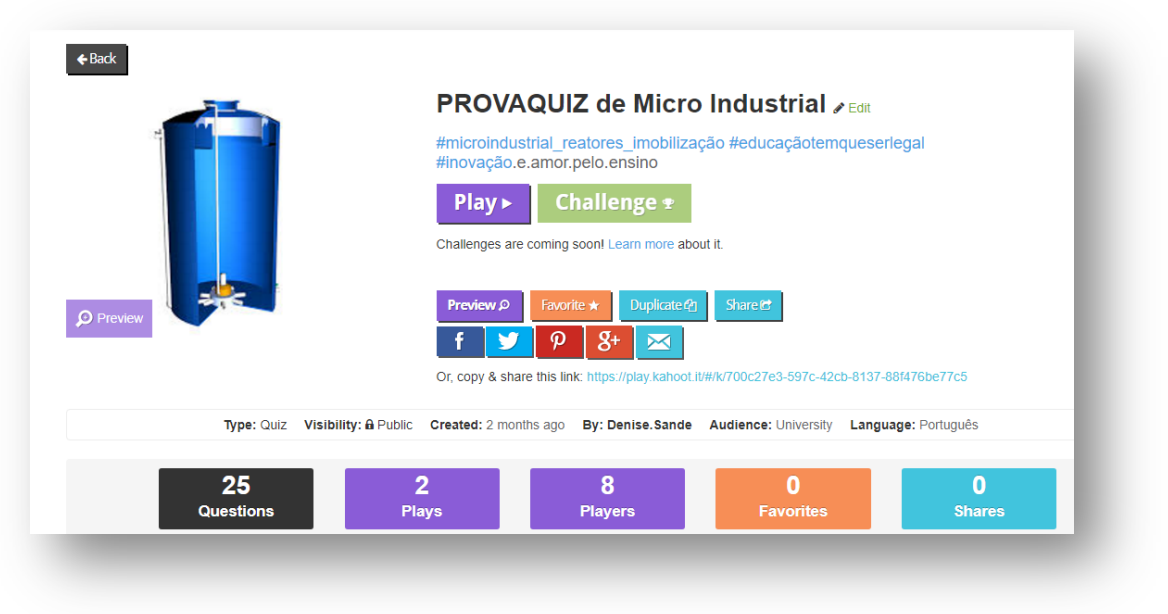

Figura 1: O kahoot do tipo quiz, intitulado PROVAQUIZ de Micro Industrial aplicado aos alunos da disciplina Microbiologia Industrial

\subsection{A aplicação do jogo}

O jogo foi criado fora da sala de aula, pelo professor, antes da data da prova. No dia da prova, o professor abriu o quiz on line e disponibilizou o link e o código de acesso para os alunos. Outro quiz da mesma modalidade já havia sido aplicado aos alunos antes desta data, assim os mesmos encontravam-se familiarizados com a metodologia. O Quiz possuía 25 questões e foi estabelecida a regra com os alunos de que cada questão do quiz valeria um ponto na nota da disciplina (a pontuação total da disciplina é de 100 pontos, assim, um quarto da nota seria avaliada pelo quiz).

A turma possuía onze alunos e eles foram divididos em 3 grupos. O quiz foi jogado no modo "team", onde as respostas são dadas por grupo e não individualmente. Nesse modo, o quiz programa um tempo de discussão entre os alunos antes de contar o tempo de resolução da questão (o tempo oficial da questão de 60, 90 ou 120 segundos, estipulado previamente, durante a criação do jogo, pelo professor).

Cada grupo escolheu um apelido e realizou o acesso ao quiz (Figura 2). Esse apelido fica disponível na tela e todos visualizam. Esse apelido é utilizado na classificação de pontos dos alunos durante o jogo. 0 jogo transcorre com a aparição de 4 telas para cada pergunta: primeiro uma tela com a pergunta, a segunda tela com a pergunta, as alternativas, e a contagem do tempo, a terceira tela com a marcação da resposta certa e com as frequências de acertos e erros daquela pergunta e a quarta tela com os resultados parciais do teste até o presente momento. Após a última pergunta, aparece um pódio com a classificação dos 3 primeiros lugares. 


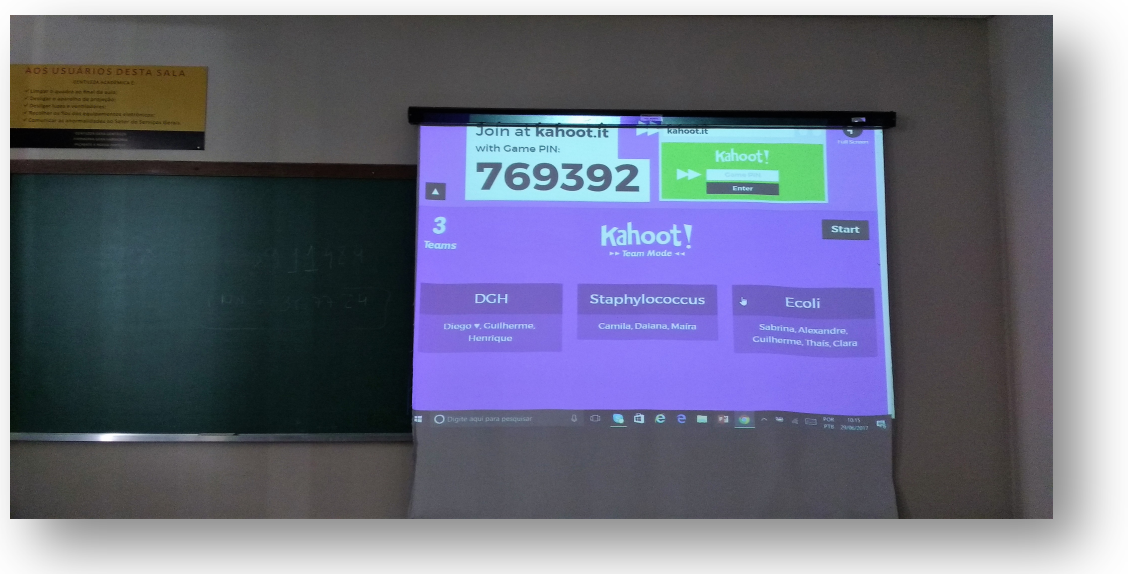

Figura 2: Projeção da tela de abertura do jogo mostrando as equipes participantes e seus componentes

\subsection{A avaliação do jogo}

Após a aplicação da prova usando o quiz, os alunos foram convidados a responder um questionário on line construído usando um formulário do GoogleDocs. Foi enviado o link do formulário (https://docs.google.com/forms/d/e/1FAlpQLSeL9pwSPukk6A34nw9nRM4sHPcCTZ9WKvPhjJHQEhZi4sk5w/viewform?usp=sf_link) para o celular dos alunos (através de um grupo no chat de conversa compartilhado com o professor) e eles responderam na sala, cada um em seu celular. No formulário foram inseridas 10 perguntas do tipo múltipla escolha:

I. As figuras ajudam ou confundem na hora de responder?

II. O kahoot é uma estratégia de avaliação que permite manter os mesmos graus de dificuldade de uma avaliação escrita (fácil, intermediário e difícil)?

III. O kahoot permite avaliar todo o conteúdo da mesma forma que uma prova escrita?

IV. O kahoot permite formular questões diretas, questões de análise e questões de raciocínio da mesma forma que uma prova escrita?

V. Fazer uma avaliação usando o kahoot, usando o "ranqueamento de acertos" e não o "ranqueamento do aplicativo" permite distribuir notas de forma justa, da mesma forma que acontece em uma prova escrita?

VI. Você acredita que consegue lembrar mais de uma questão aplicada como kahoot avaliativo ou como prova escrita?

VII. O uso do kahoot como estratégia avaliativa é mais interessante e mais atrativa do que a prova escrita?

VIII. O kahoot é uma ferramenta insuficiente para a realização de avaliação de conteúdos teóricos?

IX. Eu senti desconforto com o uso do kahoot como estratégia avaliativa por causa do "ranqueamentos de acertos" mesmo usando o "apelido" para me identificar no jogo? 
X. A gameficação inerente do kahoot, com uma competição associada, torna a avaliação usando essa estratégia mais interessante do que avaliação escrita?

XI. O tempo total do kahoot como atividade avaliativa é mais cansativo do que uma prova escrita comum?

\section{RESULTADOS E DISCUSSÃO}

Este trabalho visou determinar se a ferramenta kahoot tinha competência suficiente para ser aplicada como substituta da avaliação escrita tradicional de múltipla escolha e discursiva em turmas do ensino superior das áreas de ciência e tecnologia. Essa determinação foi feita através da análise do questionário aplicado aos alunos que vivenciaram a ferramenta e, do vídeo gravado com o depoimento dos alunos após a aplicação da ferramenta.

\subsection{Criação e aplicação do jogo}

A criação do jogo é fácil e o site é autoexplicativo. Assim, não há grandes dificuldades em inserir as questões para criar várias provas diferentes. No momento da aplicação da ferramenta, entretanto, foram percebidos alguns critérios técnicos imprescindíveis para o bom funcionamento da aplicação do quiz.

É preciso ter uma boa internet. Durante o teste realizado antes de aplicar o quiz de 25 questões dois alunos ficaram sem internet e percebeu-se que sem um sinal de internet de qualidade, fornecido gratuitamente pela universidade, não seria possível aplicar a metodologia. Para contornar esse problema, após discussão com a turma sugeriu-se o uso de redundância, ou seja, mais de um aluno deveria cadastrar o grupo e responder em consonância de tempo com o outro "administrador".

É preciso anotar o código de acesso ao jogo no quadro porque os alunos podem sair acidentalmente da tela do celular e perder o jogo. Sabendo o código, eles podem realizar uma nova entrada no jogo. Entretanto, ao entrar com o jogo já em andamento, o acesso é feito como se fosse um novo usuário com o mesmo nome do anterior e a pontuação não é acumulada. Isso significa que o jogo precisa ser realizado em modo "team", com redundância, para a pontuação seja resguardada.

O jogo tem limite de caracteres na elaboração de perguntas, no entanto, é preciso fazer alternativas menores do que o limite do jogo pois frases muito longas acabam desconfigurando ou mesmo escondendo algumas palavras das alternativas.

Só é possível usar o kahoot como método avaliativo no molde "team", ou seja, em grupo. Uma vez que a ferramenta é limitada e no pódio (última tela, a que faz a classificação dos alunos por acerto) aparecem apenas os três primeiros colocados e, não é possível saber o rendimento e ranking dos demais alunos.

\subsection{Avaliação do jogo e a percepção da prova pelos alunos}

Quando o objetivo de uma ferramenta nova é a substituição da antiga, nesse caso, um quiz on line (kahoot) no lugar de uma prova escrita (física), diferentes aspectos dessa modalidade de avaliação devem ser alcançados pela modalidade a ser implementada. Assim, a percepção de diferentes aspectos da ferramenta kahoot foram avaliados pelos alunos através do questionário 
e, os resultados podem ser apreciados na Tabela 1. De uma forma geral pode-se perceber que os alunos vislumbram a viabilidade da ferramenta kahoot como substituto de uma avaliação tradicional, uma vez que a maioria dos alunos acreditam que a ferramenta permite gerar diferentes graus de dificuldade (fácil, moderado e difícil) esperados em um processo de avaliação (60\%). Eles também perceberam que o kahoot permite construir questões de raciocínio direto, mas também questões que demandam análise e raciocínio (70\%). Todos os alunos acreditam na competência do quiz em gerar notas justas a partir da quantificação dos acertos.

No tocante à capacidade de avaliar o conteúdo, a maioria dos alunos (80\%) afirmou que a ferramenta é eficiente nesse processo. Entretanto, os alunos ficaram divididos quanto ao uso apenas dessa ferramenta para a avaliação do conteúdo teórico (Tabela 1). Essa conclusão foi reforçada nos depoimentos, quando um aluno relatou que via a necessidade de complementar a ferramenta kahoot com uma prova aplicada no mesmo dia com questões discursivas.

Os alunos avaliaram a ferramenta kahoot como mais interessante do que a prova tradicional escrita (80\%) e atribuíram isso à competitividade intrínseca do jogo (Tabela 1). Isso ressalta a importância de inserir aspectos de jogos, como a gamificação, no contexto acadêmico tanto de ensino-aprendizagem como de avaliação. Dellos (2015) corrobora com essa ideia e ressalta que essa competitividade do jogo torna a experiência do aprendizado mais valiosa para os alunos.

Tabela 1: Percepção dos alunos sobre as competências da ferramenta kahoot como método avaliativo.

\begin{tabular}{c|c|c}
\hline Perguntas & Sim (\%) & Não (\%) \\
\hline $\begin{array}{c}\text { Permite manter os } \\
\text { graus de dificuldade } \\
\text { (fácil, moderado e } \\
\text { difícil) }\end{array}$ & 60 & 40 \\
$\begin{array}{c}\text { Permite avaliar } \\
\text { questões diretas, de } \\
\text { análise e de } \\
\text { raciocínio }\end{array}$ & 70 & 30 \\
$\begin{array}{c}\text { Permite uma nota } \\
\text { justa usando a } \\
\text { classificação de } \\
\text { acertos }\end{array}$ & 100 & 0 \\
$\begin{array}{c}\text { Permite avaliar } \\
\text { todo o conteúdo }\end{array}$ & 80 & 20 \\
$\begin{array}{c}\text { É uma ferramenta } \\
\text { insuficiente para } \\
\text { avaliar o conteúdo } \\
\text { teórico }\end{array}$ & & \\
$\begin{array}{c}\text { Permite uma } \\
\text { avaliação mais } \\
\text { interessante e } \\
\text { atrativa }\end{array}$ & 50 & 20 \\
$\begin{array}{c}\text { Por ser um jogo e } \\
\text { ter competição é } \\
\text { mais interessante e } \\
\text { atrativa }\end{array}$ & 80 & \\
\hline
\end{tabular}

Alguns autores, como Alves \& Maciel (2014), preconizam que elementos dos jogos, tais como as metáforas e abstrações, fazem parte do conjunto de elementos que visa criar uma atmosfera relacionada ao jogo. Observando a Tabela 1 é possível perceber que, segundo a 
maioria dos alunos (80\%), a adição de figuras no "quiz" parece não ter influenciado o raciocínio dos alunos. Isso parece estar relacionado ao pouco tempo (60 a 120 segundos) que eles têm para responder as questões e no seu impacto diante da nota. Assim, o foco na pergunta e alternativas precisa ser mantido para que haja tempo hábil de criar um conceito, compartilhar com o grupo e selecionar uma resposta final. Na estratégia de uso do kahoot como ferramenta avaliativas, esse elemento parece não ter sido tão importante, dentro do contexto do jogo aplicado.

Quando são aplicados jogos on line, é necessário também preocupar-se com a exposição que os alunos serão submetidos. Nesse jogo, kahoot, os alunos entram com um apelido e este aparece na tela (Figura 2), permitindo a identificação dos grupos e alunos que estão jogando naquele momento. Assim, uma das perguntas visava avaliar se algum aluno sentia-se prejudicado ou constrangido por "aparecer" como um apelido na tela do jogo. A maioria dos alunos (Figura 3) não sentiu desconforto com esse quesito. Esse é um fator importante uma vez que a prova escrita é totalmente individualizada e não expõe os alunos à essas situações.

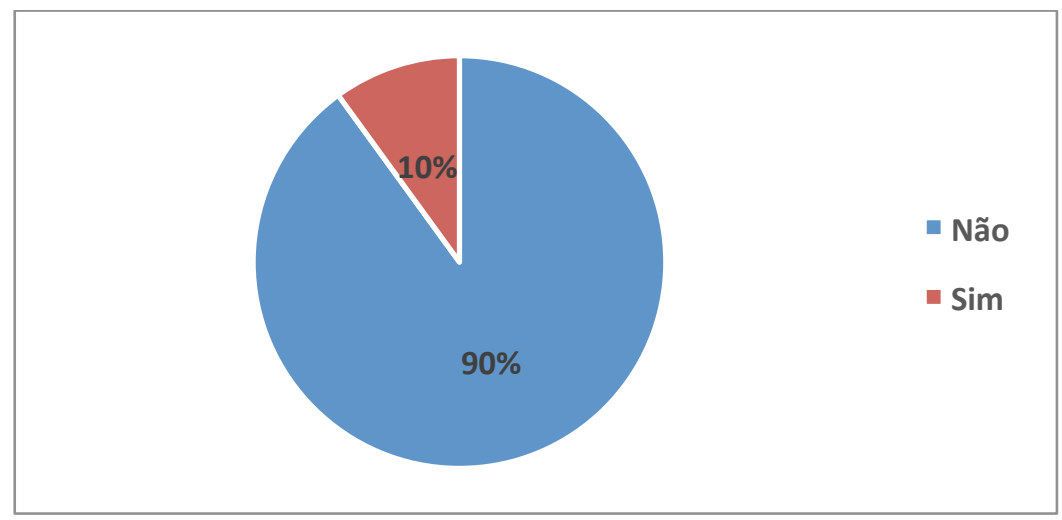

Figura 3: Resposta dos alunos à pergunta "Eu senti desconforto com o uso do kahoot como estratégia avaliativa por causa da classificação de acertos mesmo usando o apelido para identificar-me no jogo? ".

(Fonte: arquivo pessoal, 2017)

A observação da Figura 4 permite inferir que os tempos utilizados na avaliação usando o kahoot foram suficientes, na percepção dos alunos. O tempo para responder às perguntas deve ser calculado em função do tipo de construção da pergunta, bem como em relação ao grau de dificuldade da questão (Costa et al., 2017). No entanto, por se tratar de um jogo, o tempo não deve ser muito longo para que a atividade não perca sua capacidade lúdica. $O$ tempo não deve ser muito curto também, pois, isso poderia trazer um resultado errôneo e que não refletiria a realidade do aluno uma vez que este poderia ter o conhecimento para responder à questão, mas uma formulação incorreta do tempo não permitiria que ele aplicasse o seu conhecimento na resolução. 


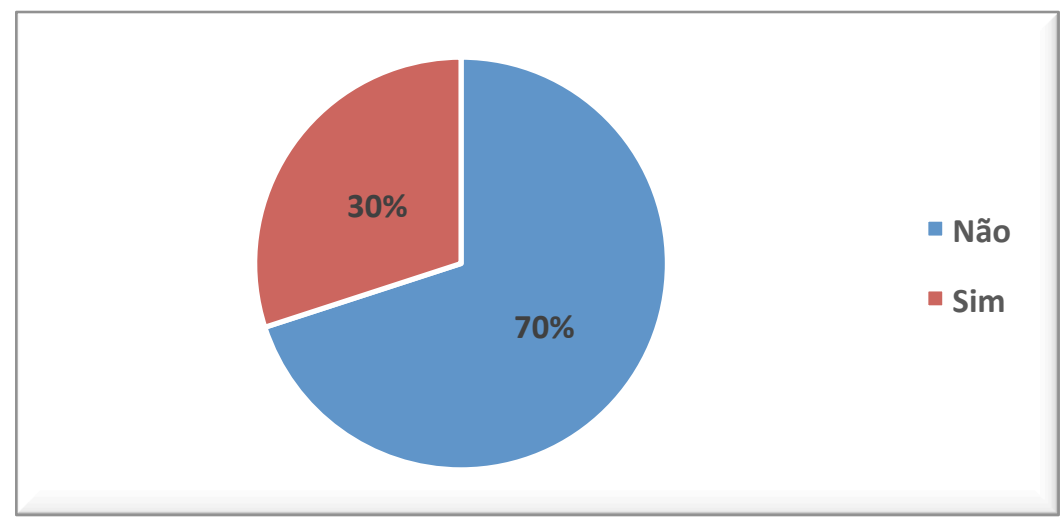

Figura 4: Resposta dos alunos à pergunta "O tempo total do kahoot como atividade avaliativa é mais cansativo do que uma prova escrita comum?".

(Fonte: arquivo pessoal, 2017)

A aprendizagem é um processo diferente entre os alunos. Por isso, o uso de diversidade de estratégias durante as aulas é imprescindível para que o máximo de alunos consiga compreender os conteúdos abordados. Também nesse aspecto a ferramenta kahoot demonstrou ser útil. A Figura 5 permite inferir a capacidade de memorização associada à ferramenta kahoot. A maioria dos alunos (60\%) afirmou que lembraria mais de uma questão aplicada como kahoot do que como prova tradicional em função da competição associada ao jogo (40\%) e em função da angústia gerada pelo pouco tempo para responder à questão (20\%). Apenas uma minoria acredita que a ferramenta é tão eficiente quanto o ensino tradicional em fazê-los lembrar do conteúdo e nenhum aluno afirmou que lembraria mais do conteúdo de uma questão se ele fosse aplicado apenas como uma prova escrita tradicional. Isso ressalta que no contexto do kahoot os alunos retêm melhor os conhecimentos do que no formato da prova tradicional, de acordo com a percepção dos próprios alunos.

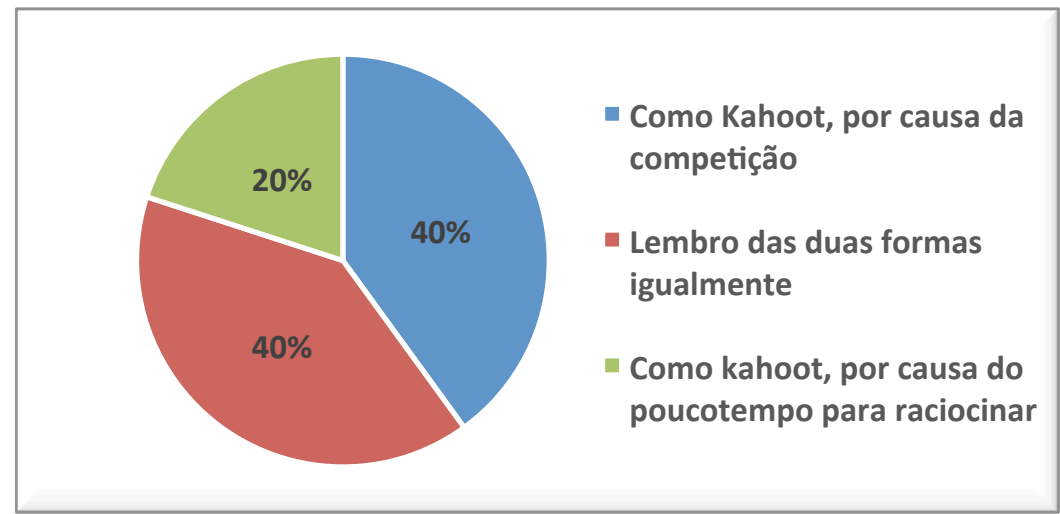

Figura 5: Resposta dos alunos à pergunta "Você acredita que consegue lembrar mais de uma questão aplicada como kahoot avaliativo ou como prova escrita?".

(Fonte: arquivo pessoal, 2017) 


\section{CONCLUSÃO}

A ferramenta kahoot foi aplicada como instrumento avaliativo dentro da disciplina Microbiologia Industrial e mostrou-se muito efetiva como substituto da prova tradicional, na percepção dos estudantes. Segundo a maioria deles (60\%) a ferramenta permite uma avaliação com diferentes níveis de complexidade e permite uma maior memorização e entendimento dos conteúdos do que a prova tradicional. A maioria dos estudantes $(80 \%)$ concluiu que essa ferramenta permite avaliar todo o conteúdo teórico da disciplina de forma mais atraente e competitiva, criando um grande estímulo. Todos os alunos concluíram que a ferramenta tem um sistema de pontuação justo e permite obter notas de maneira justa.

A partir da observação do comportamento dos alunos foi possível perceber que além da pontuação referente à prova é conveniente ter um prêmio envolvido com a aplicação da atividade, como uma nota bônus para o primeiro lugar ou premiação com chocolate, pois assim há maior competição e estímulo aos acertos.

Percebeu-se que a técnica apresenta algumas limitações de uso, entretanto, o jogo causou um estímulo nos alunos deixando o processo avaliativo mais atraente e o aprendizado mais duradouro. Desta forma, a percepção e interesse dos alunos foi positiva diante da experiência o que a coloca em um patamar de estratégia válida para o ensino e avaliação da disciplina Microbiologia industrial.

\section{REFERÊNCIAS}

Bottentuit Júnior, J. B. (2012) Do computador ao tablet: vantagens pedagógicas na utilização de dispositivos móveis na educação. Revista educaonline, 6(1), 125-149.

Cavalcanti, E. L. D, \& Soares, M. H. F. B. (2009) O uso do jogo de roles (roleplaying game) como estratégia de discussão e avaliação do conhecimento químico. Revista Electrónica de Enseñanza de las Ciencias, 8(1), 255-282.

Coil, D. A., Ettinger, C. L., \& Eisen, J. A. (2017) Gut Check: The evolution of an educational board game. PLOS Biology, 15(4), e2001984.

Costa, C. H. C., Dantas Filho, F. F., \& Moita, F. M. G. S. C. (2009) Marvinsketch e kahoot como ferramentas no ensino de isomeria. HOLOS, 1, 31-43.

Dellos, R. (2015) Kahoot! A digital game resource for learning. International Journal of Instructional Technology and Distance Learning, 12(4), 49-52.

Martinez, E. R. M, Fujihara, R. T., \& Martins, C. (2008) Show da genética: um jogo interativo para o ensino de genética. Genética na escola, 3(2), 24-27.

Mitre, S. M., Siqueira-Batista, R., Girardi-de-Mendonça, J. M., Morais-Pinto, N. M., Meirelles, C. A. B., Pinto-Porto, C., Moreira, T., \& Hoffmann, L. M. A. (2008) Metodologias ativas de ensinoaprendizagem na formação profissional em saúde: debates atuais. Ciência \& Saúde Coletiva, 13(2), 2133-2144.

Tardif, M. (2011). Saberes docentes e formação profissional (12. ed.). Petrópolis, RJ: Vozes.

Van Nuland, S. E., Roach, V. A., Wilson, T. D., \& Belliveau, D. J. (2015) Head to head: the role of academic competition in undergraduate. Anatomical Sciences Education, 8, 404-412. 